\title{
Effects of Artificial Light Source and Light Quality on the Growth of Two Species of Microalgae
}

\author{
Hongwu Cui, Fanping Meng*, Yongfu Li, Yuejie Wang, Weiyan Duan
}

Key Laboratory of Marine Environment and Ecology, Ministry of Education, College of Environmental Science and Engineering, Ocean University of China, Qingdao, China

\section{Email address:}

hongwuc@tom.com (Hongwu Cui), fanpingm@tom.com (Fanping Meng), lyf_qingdao@126.com (Yongfu Li), wyuejie@163.com (Yuejie Wang), duanweiyan@163.com (Weiyan Duan)

${ }^{*}$ Corresponding author

\section{To cite this article:}

Hongwu Cui, Fanping Meng, Yongfu Li, Yuejie Wang, Weiyan Duan. Effects of Artificial Light Source and Light Quality on the Growth of Two Species of Microalgae. Science Discovery. Vol. 4, No. 2, 2016, pp. 129-136. doi: 10.11648/j.sd.20160402.22

Received: May 3, 2016; Accepted: May 17, 2016; Published: May 18, 2016

\begin{abstract}
The effects of different kinds of artificial light source and light quality on two species of microalgae (Chlorella vulgaris and Dunaliella salina) of high resistance of acid, heat and $\mathrm{CO}_{2}$ concentration were investigated in this research, 9 light qualities (LR, LB, LW, FL, LR+LB, LW+LR, LW+LB, FW+LR and FW+LB) were set up with 4 kinds of light source (LED-Red (LR), LED-Blue (LB), LED-White (LW)) and Fluorescent lamp white (FW) to cultivate microalgae, while biological parameters and $P-I$ curves were measured and drawn to evaluate the effect of light source and light quality on growth characteristics of microalgae, respectively. Results showed that $\overline{P_{m}}$ of two microalgae were observed under the illumination of LED-W when 4 kinds of light source were employed. At the end of cultivation, maximum biomass of $C$. vulgaris were observed under the illumination of light qualities LW+LB, which were $0.19 \mathrm{~g} \cdot \mathrm{L}^{-1}$, however, the highest growth rate was obtained under the illumination of light quality LB by $D$. salina, which was 1.5 times as much as that in LR treatment. In addition, the nonuniformity between photosynthetic pigments content and $\mu$ along with the changed light quality indicated that the variation of photosynthetic pigments content may not be the main cause of microalgae growth regulated by light quality, while the light absorption of living cells might be the primary reason affecting the growth of microalgae.
\end{abstract}

Keywords: Light Source, Light Quality, Light Emitting Diode (LED), Growth Characteristics, Photosynthetic Oxygen eVolution

\section{人工光源和光质对 2 株微藻生长的影响}

\author{
崔鸿武, 孟范平*, 李永富, 王曰杰, 段伟艳 \\ 中国海洋大学海洋环境与生态教育部重点实验室, 环境科学与工程学院, 青岛, 中国 \\ 邮箱 \\ hongwuc@tom. com（崔鸿武）, fanpingm@tom. com（孟范平）, 1yf_qingdao@126. com（李永富）, wyuejie@163. com(王曰杰), \\ duanweiyan@163. com（段伟艳）
}

摘要：为探究不同人工光源及光质对 2 株耐高温、耐酸性、耐高浓度 $\mathrm{CO}_{2}$ 特性的绿藻普通小球藻 (ChIorella vulgaris) 和盐生杜氏藻 (Dunaliella salina) 生长的影响, 本研究采用4种人工光源（LED红光（LR）、LED蓝光（LB）、LED白光 $(L W))$ 和苂光灯全光谱白光 $(F W)$ 营造的9种光质（LR、LB、LW、FL、LR+LB、LW+LR、 $L W+L B 、 F W+L R$ 以及FW+LB）对2 株微藻进行连续培养, 通过多种生物学指标测定以及 $P-I$ 曲线绘制等方法评价了不同光源、光质对微藻生长特性的影响。 结果表明, 使用 4 种光源培养微藻, 最大光合放氧速率均出现在LED-W光源下; 至培养结束时, 普通小球藻以 $\mathrm{LW}+\mathrm{LB}$ 光质 照射下的藻生物量最高, 为 $0.19 \mathrm{~g} \cdot \mathrm{L}^{-1}$, 而在盐生杜氏藻培养期间, $\mathrm{LB}$ 照射下的藻生物量一直高于其他光质, 为LR的 1.5 
倍; 另外, 光合色素含量与 $\mu$ 值随光质变化的非一致性说明光合色素变化可能不是光质调控微藻生长的主要原因, 而 藻细胞的活体光吸收可能是影响微藻生长的主要因素。

关键词: 光源, 光质, LED, 生长特性, 光合放氧

\section{1. 引言}

微藻在生物固碳、生物柴油、污水处理、水产养殖以 及食品等领域有着广阔的应用前景。构建适于微藻生长的 光生物反应器 (PBR) 是实现微藻快速培养、满足相关产业 需要的重要课题 $[1]$ 。目前, 多采用敞开式大池 $(O P)$ 和封 闭式光生物反应器 (PBR) 两种形式用于微藻养殖 [2]。与前 者相比, 封闭式PBR因具有培养条件稳定、可无菌操作、 易于高密度培养等优点, 成为微藻生物技术所需工程设备 的发展方向 [3]。

影响微藻生长的原因很多, 其中, 光照是影响封闭 式PBR中微藻生长速率的关键因素之一。因此, 研究光照 作用及应用适当的光照技术培养微藻, 加快其生长繁殖, 调节其营养成分, 是提高其产量和质量的重要途径 [4], 在封闭式PBR 设计时应首先考虑。近年来, 发光二极管 (1ight emitting diode, LED) 作为一种新型冷光源因具 有电源电压低、投光远、工作温度范围广、节能高效、 稳定性强、多色、安全、高亮度、长寿命、体积小、发 热少、响应快、无频闪、无污染、无辐射、无故障等优 点 [5], 已经在生态照明领域展现出非常广阔的应用前景 [6]。柔性LED灯带因防水性好、几乎可以任意角度弯曲, 有望成为封闭式PBR的新型内置光源。另外, 根据微藻的 光生态学, 光合作用要求光源的峰值波长与微藻活体吸 收峰值相对应 [1]。单色蓝光或红光有利于提高光能利用 率 [7], 如蛋白核小球藻 (Chlorella pyrenoidosa) [8] 在 蓝光下的生长效果最佳。然而, 也有研究表明, 微藻需 要一些微弱但必须的波长成分进行生长调节, 因此, 除 蓝光和红光外的其他波段光对藻种也是有益的, 例如, 中胁骨条藻 (Skeletonema costatum) [9] 和中华盒形藻 (Biddulphia sinensis) [10] 在荧光灯下生长情况优于 单色蓝光、单色红光或红蓝组合光; 沈银武等 [11]研究 蓝藻中华植水藻 (Richelia sinica (Shen)) 时发现, 白 光和黄光对微藻生长最有利, 绿光和红光次之, 蓝光下 较慢。这体现了光质对微藻生长影响的复杂性。因此, 应根据实验确定适于不同藻种生长的光源类型及光质组 成。

本研究以篎选出的耐高温 $\left(30^{\circ} \mathrm{C}\right)$ 、耐酸性 $(\mathrm{pH} 4.0)$ 、 耐高浓度 $\mathrm{CO}_{2}\left(5^{\sim} 15 \%\right)$ 特性的 2 种微藻, 即普通小球藻 $(C$. vulgaris) 和盐生杜氏藻 (Dunaliella salina) 为供试藻 种, 探究红光LED灯带 (LED-R)、蓝光LED灯带 (LED-B) 、 白光LED灯带 (LED-W) 和苂光灯 (FL) 这 4 种人工光源对微藻 生长的影响, 分析光质变化引起微藻生长速率差异的可能 原因, 以便为封闭式PBR中的光源设计提供依据。

\section{2. 材料与方法}

\section{1 . 材料}

\subsection{1. 供试藻种}

普通小球藻 (C. vulgaris) 购自中科院水生生物研究 所; 盐生杜氏藻 (D. salina) 由中国海洋大学微藻种质库 提供。
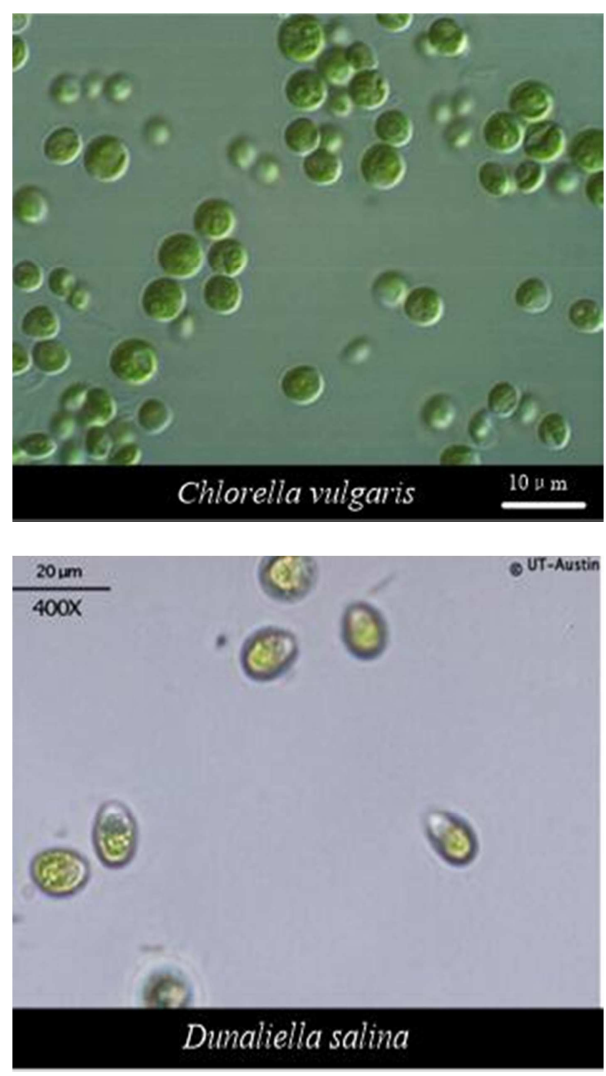

图12种受试微藻的细胞形态。

\subsection{2. 培养基}

普通小球藻 (C. vulgaris) 与盐生杜氏藻 (D. salina) 分别采用SE和 $f / 2$ 培养基培养。

SE培养基每升蒸馏水中含有以下成分： $250 \mathrm{mg} \mathrm{NaNO}$ 、 $75 \mathrm{mg} \mathrm{K} \mathrm{HPO}_{4} 、 75 \mathrm{mg} \mathrm{MgSO}_{4} \cdot 7 \mathrm{H}_{2} \mathrm{O}$ 、 $25 \mathrm{mg} \mathrm{CaCl} \mathrm{Cl}_{2} \cdot 2 \mathrm{H}_{2} \mathrm{O}$ 、 $175 \mathrm{mg} \mathrm{KH} \mathrm{PO}_{4} 、 25 \mathrm{mg} \mathrm{NaCl} 、 5 \mathrm{mg} \mathrm{FeCl}{ }_{3} \cdot 6 \mathrm{H}_{2} \mathrm{O} 、 2.86 \mathrm{mg}$ $\mathrm{H}_{3} \mathrm{BO}_{3} 、 1.86 \mathrm{mg} \mathrm{MnCl}_{2} \cdot 4 \mathrm{H}_{2} \mathrm{O} 、 0.22 \mathrm{mg} \mathrm{ZnSO} \cdot 7 \mathrm{H}_{2} \mathrm{O} 、 0.39$ mg $\mathrm{Na}_{2} \mathrm{MoO}_{4} \cdot 2 \mathrm{H}_{2} \mathrm{O} 、 0.049 \mathrm{mg} \mathrm{Co}\left(\mathrm{NO}_{3}\right) \cdot 6 \mathrm{H}_{2} \mathrm{O} 、 0.079 \mathrm{mg}$ $\mathrm{CuSO}_{4} \cdot 5 \mathrm{H}_{2} \mathrm{O} 、 1 \mathrm{~mL}$ EDTA $・ \mathrm{Fe} （$ 将0.901g FeCl $\mathrm{g}_{3} \cdot 6 \mathrm{H}_{2}$ O溶于 $10 \mathrm{~mL}$ 的 $1 \mathrm{~mol} \cdot \mathrm{L}^{-1}$ 稀盐酸中, 再与 $10 \mathrm{~mL} 0.1 \mathrm{~mol} \cdot \mathrm{L}^{-1}$ 的 EDTA- $\mathrm{Na}_{2}$ 溶液混合, 加入蒸馏水稀释至 $1 \mathrm{~L}$ )、 $40 \mathrm{~mL}$ 土壤 
浸出液 (将 $200 \mathrm{~g}$ 避光晾干的未施肥花园土溶于 $1 \mathrm{~L}$ 去离子 水中, 水浴煮沸 $3 \mathrm{~h}$, 冷却沉淀 $24 \mathrm{~h}$, 重复煮沸 3 次。过滤 取上清液, 高压灭菌后 $4^{\circ} \mathrm{C}$ 冷藏保存备用)。培养基在使用 前于 $120^{\circ} \mathrm{C}$ 下灭菌 $20 \mathrm{~min}$ 。

$\mathrm{f} / 2$ 培养基每升海水中含有以下成分： $74.8 \mathrm{mg} \mathrm{NaNO}$ 、 $4.4 \mathrm{mg} \mathrm{NaH} \mathrm{PO}_{4} 、 0.023 \mathrm{mg} \mathrm{ZnSO}_{4} \cdot 4 \mathrm{H}_{2} \mathrm{O} 、 0.012 \mathrm{mg}$ $\mathrm{CoCl}_{2} \bullet 6 \mathrm{H}_{2} \mathrm{O} 、 3.9 \mathrm{mg} \mathrm{FeC} \mathrm{H}_{5} \mathrm{O}_{7} \cdot 5 \mathrm{H}_{2} \mathrm{O}$ 、 $4.35 \mathrm{mg} \mathrm{Na} 2-$ EDTA、 $0.178 \mathrm{mg} \mathrm{MnCl} l_{2} \cdot 4 \mathrm{H}_{2} \mathrm{O} 、 0.010 \mathrm{mg} \mathrm{CuSO}{ }_{4} \cdot 5 \mathrm{H}_{2} 0 、 0.0073 \mathrm{mg}$ $\mathrm{Na}_{2} \mathrm{MoO}_{4} \cdot 2 \mathrm{H}_{2} \mathrm{O} 、 0.0005 \mathrm{mg}$ 维生素B $\mathrm{B}_{12} 、 0.100 \mathrm{mg}$ 维生素 $\mathrm{B}_{1}$ 以及 $0.0005 \mathrm{mg}$ 维生素H。

\subsection{3. 试剂}

丙酮及培养基配制所用的试剂均为国产分析纯。SE 培养基用去离子水配制, $\mathrm{f} / 2$ 培养基所用海水取自青岛石 老人近岸海域, 经 $0.45 \mu \mathrm{m}$ 混合纤维滤膜抽滤, 高温灭菌 后冷却备用。

\subsection{4. 仪器}

(1) 人工光源

LXHL-PM09型LED-R、LED-B、LED-W和F25T8/TL 950 型FL由荷兰Philips公司生产, 分别用于提供LED红光 (LR)、 LED蓝光 (LB)、 $L E D$ 白光 $(\mathrm{LW})$ 和荧光灯全光谱白光 $(F W)$ 。各 光源的主要光学参数见表 1 。

表 14 种光源的光学参数。

\begin{tabular}{llll}
\hline 光源 & 波峰 $(\mathrm{nm})$ & 波段 $(\mathrm{nm})$ & $\begin{array}{l}\text { 发射光强 } \\
\left(\boldsymbol{\mu} \mathrm{mol} \cdot \mathrm{m}^{-2} \cdot \mathrm{s}^{-1}\right)\end{array}$ \\
\hline LED-R & 627 & $620^{\sim} 645$ & $30 \pm 3$ \\
LED-B & 470 & $460^{\sim} 490$ & $30 \pm 3$ \\
LED-W & $627,470,530$ & $620^{\sim} 645,520^{\sim} 550,60^{\sim} 490$ & $60 \pm 3$ \\
FL & - & $400^{\sim} 700$ & $30 \pm 3$ \\
\hline
\end{tabular}

(2) 其他仪器

CXZ智能型光照培养箱(宁波江南仪器厂) ; LDZX-50KBS 型高压蒸汽灭菌锅 (上海申安医疗器械厂) ; 3415F型光量子 计 (美国Spectrum公司); UNICOUV-2600型紫外可见分光光 度计 (优尼科 (上海) 有限公司); T6 型紫外可见分光光度计 (北京普析通用仪器有限责任公司); SX716型溶解氧测量仪 (极谱型D0电极, 上海三信仪表厂); Nikon YS2-H型电子显 微镜 (日本Nikon公司); Freezone2.5L型真空冷冻干燥机 (美国Labconco公司); XW-80A型旋浴混合器 (宁波新芝生物 科技股份有限公司); GM- $0.33 \mathrm{~A}$ 型隔膜真空原 (天津市津腾 实验设备有限公司) ; DHG-9030A型电热恒温鼓风干燥箱 (上 海一恒科学仪器有限公司)。

\section{2. 方法}

\subsection{1. 微藻在 4 种光源下的 $P-I$ 曲线绘制}

取经过FL预培养至指数生长期的 2 种微藻, 在 LED $-R$ 、 LED-B、 LED-W和FL四种光源, 不同光量子通量密度下进 行光合放氧测定, 绘制 $P-I$ 曲线。步骤如下: 将处于指数 生长期的藻细胞转移到 $100 \mathrm{~mL}$ 碘量瓶中, 在与培养温度
相同的环境中暗处培养 $2 \mathrm{~h}$, 测定呼吸作用的耗氧速率。 然后, 在持续照光条件下进行净光合放氧速率测定。期 间, 通过控制LED灯的数量或者碘量瓶与苂光灯管的距离, 营造从 20 至 $120 \mathrm{\mu} \mathrm{mol} \cdot \mathrm{m}^{-2} \cdot \mathrm{s}^{-1}$ 的 6 个光量子通量梯度。总 光合放氧速率 $\left(\mu \mathrm{mol} \mathrm{O}_{2} \cdot \mathrm{g}^{-1} \cdot \mathrm{h}^{-1}\right)$ 为净光合放氧速率与呼 吸耗氧速率之和。最大总光合放氧速率 $\left(P_{m}\right)$ 及其他参数 由Han模型计算 [12]:

$$
\begin{aligned}
& \mathrm{P}=\mathrm{N} \frac{\sigma_{\mathrm{PSII}} \mathrm{I}}{1+\sigma_{\mathrm{PSII}} \tau+\mathrm{K}\left(\sigma_{\mathrm{PSII}} \mathrm{I}\right)^{2} \tau} \\
& \overline{\mathrm{P}}_{\mathrm{m}}=\frac{\mathrm{N}}{\tau+2 \sqrt{\mathrm{K} \tau}} \\
& \overline{\mathrm{I}}_{\mathrm{k}}=\frac{1}{\sigma_{\mathrm{PSII}}(\tau+2 \sqrt{\mathrm{K} \tau})} \\
& \mathrm{S}_{0}=\mathrm{N} \sigma_{\mathrm{PSII}} \\
& \overline{\mathrm{I}}_{\mathrm{m}}=\frac{1}{\sigma_{\mathrm{PSII}} \sqrt{\mathrm{K} \tau}}
\end{aligned}
$$

式中, $P$ 为总光合放氧速率 $\left(\mu \mathrm{mol} \quad \mathrm{O}_{2} \cdot \mathrm{g}^{-1} \cdot \mathrm{h}^{-1}\right), \quad \overline{P_{m}}$ 为 最大光合作用速率 $\left(\mu \mathrm{mol} \quad \mathrm{O}_{2} \cdot \mathrm{g}^{-1} \cdot \mathrm{h}^{-1}\right), I_{k}$ 为半饱和光强 $\left(\mu \mathrm{mol} \cdot \mathrm{m}^{-2} \cdot \mathrm{s}^{-1}\right), S_{0}$ 为初始斜率 $\left(\mu \mathrm{mol} \mathrm{O}_{2} \cdot \mathrm{g}^{-1} \cdot \mathrm{h}^{-1} \cdot\left(\mu \mathrm{mol} \cdot \mathrm{m}^{-2} \cdot \mathrm{s}\right.\right.$ $\left.\left.{ }^{-1}\right)^{-1}\right), \quad \overline{I_{m}}$ 为最优光强 $\left(\mu \mathrm{mol} \cdot \mathrm{m}^{-2} \cdot \mathrm{s}^{-1}\right), \quad N$ 为单位叶绿素中 光合单位的数量 $\left(\mathrm{mol} \mathrm{O}_{2} \cdot(\mathrm{mg} \mathrm{Chl}-\mathrm{a})^{-1}\right), \tau$ 为电子传递链 的周转时间 (s), $\sigma_{P S I I}$ 为PSII的吸收截面积 $\left(\mathrm{m}^{2} \cdot\right.$ quantum $^{-1}$ ), $K$ 为光破坏速率常数/修复常数的比值, 反映了光抑制实现 的程度, $K$ 值越大, 说明光抑制的程度越强。通过最小二 乘法进行曲线 (式 (1) 的拟合优度检验, 求得的可决系数 $\mathrm{R}$ 平均值为 0.99 。

\subsection{2. 光质对微藻生长的影响研究}

在恒温培养箱, FL照射下对2株微藻进行预培养, 至 指数生长期后定量接种于装有 $300 \mathrm{~mL}$ 新鲜培养基的 $500 \mathrm{~mL}$ 锥形瓶中, 初始接种藻细胞密度为普通小球藻 $1.5 \times 10^{6}$ cells $\cdot \mathrm{mL}^{-1}$ 和盐生杜氏藻 $0.75 \times 10^{6} \mathrm{ce} 11 \mathrm{~s} \cdot \mathrm{mL}^{-1}$ 。 $25^{\circ} \mathrm{C}$ 条件 下, 将微藻置于 4 种光源营造的 9 种光质 (光量子通量密度 均为 $\left.60 \mathrm{\mu} \mathrm{mol} \cdot \mathrm{m}^{-2} \cdot \mathrm{s}^{-1}\right)$ 条件下培养。

9 种光质中，除LW、LB、LR和FW外，还包括以下5种混 合光质: $L R$ 与 $L B$ 混合光质 $(L R+L B)$ 、 $L W$ 与 $L R$ 混合光质 $(L W+L R) 、 L W$ 与 $L B$ 混合光质 $(L W+L B) 、 F W$ 与 $L R$ 混合光质 $(F W+L R) 、 F W$ 与B 混合光质 $(F W+L B)$, 分别按光量子通量密 度 $1: 1$ 由对应的两种光质混合而成。

各实验组设置三个平行, 采用摇瓶批次培养模式, 在 恒温培养箱中进行。

\section{2. 3. 微藻生物量测定}

采用细胞干重法[13]测定。取 $10 \mathrm{~mL}$ 藻液, 用 $0.45 \mu \mathrm{m}$ 混合纤维滤膜（预先在 $70^{\circ} \mathrm{C}$ 烘干至恒重）抽滤。然后用蒸 馏水冲洗 3 次, 于 $70^{\circ} \mathrm{C}$ 下烘至恒重, 用感量 $0.1 \mathrm{mg}$ 天平准 确称重。每组均进行滤膜空白校正。 


\section{2. 4. 藻细胞密度测定与比生长速率计算}

藻细胞密度: 固定时间取样, 用血球计数板个体计数 法测定, 平行三次。

指数生长期微藻的比生长速率按式 (6) 计算:

$$
\mu=\frac{\ln \mathrm{N}_{\mathrm{t}}-\ln \mathrm{N}_{0}}{\mathrm{t}}
$$

式中, $\mu$ 为比生长速率 $\left(\mathrm{d}^{-1}\right) ; N_{t}$ 为 $t(\mathrm{~d})$ 时刻的藻细胞 数量 $\left(\mathrm{ce} 11 \mathrm{~s} \cdot \mathrm{mL}^{-1}\right), N_{0}$ 为初始藻细胞数量 $\left(\mathrm{ce} 11 \mathrm{~s} \cdot \mathrm{mL}^{-1}\right)$ 。

\section{2. 5. 藻细胞活体吸收谱}

用UNICO UV-2600型紫外可见分光光度计测定藻细胞 活体吸收谱。以新鲜培养基为空白扫描基线, 然后在PAR 波段 $\left(400^{\sim} 700 \mathrm{~nm}\right)$ 内测试试样。光束为直射光, 光谱带宽 $1 \mathrm{~nm}$, 扫描速率 $5 \mathrm{~nm} \cdot \mathrm{s}^{-1}$ 。

\subsection{6. 光合色素分析}

采用Sükran等 [14] 的方法测定叶绿素a (Chl-a) 、叶 绿素b (Ch1-b) 、类胡萝卜素 (Car) 的含量。收集 $20^{\sim} 60 \mathrm{~mL}$ 藻液, $20^{\circ} \mathrm{C}$ 下 $3000 \mathrm{rpm}$ 离心 $10 \mathrm{~min}$, 用蒸馏水清洗三次脱 盐, 吸水纸吸干表层水分, 称取湿藻泥的重量, 以固液比 $50 \mathrm{~mL} \cdot \mathrm{g}^{-1}(\mathrm{fw})$ 加入 $90 \%$ 丙酮, 漩涡混合 $1 \mathrm{~min}, 4^{\circ} \mathrm{C}$ 避光冷藏 过夜。然后经双层纱布过滤后5000 rpm离心10 min, 用紫 外可见分光光度计读取上清液在 $450 \mathrm{~nm}, 630 \mathrm{~nm}, 647 \mathrm{~nm}$, $664 \mathrm{~nm}, 691 \mathrm{~nm}$ 处的吸光度, 按Ritchie[15]的公式计算 Ch1 $-a$ 和Ch1- $b$ 含量, 按Jensen[16]的方法计算Car含量。

Ch1-a $\left(\mathrm{mg} \cdot \mathrm{L}^{-1}\right)=-0.3319 \mathrm{~A}_{630}-1.7485 \mathrm{~A}_{647}+11.9442 \mathrm{~A}_{664}-1.430$

Ch1 $-b\left(\mathrm{mg} \cdot \mathrm{L}^{-1}\right)=-1.2825 \mathrm{~A}_{630}+19.8893 \mathrm{~A}_{647}-4.8860 \mathrm{~A}_{66 t}-2.341$

$$
\operatorname{Car}\left(\mathrm{mg} \cdot \mathrm{L}^{-1}\right)=\mathrm{A}_{450} \times 10000 / 2500
$$

\section{3. 数据统计与分析}

每种处理下的 $\mu$ 值、光合色素含量、P值分别用三次 平行测定结果的平均值士标准偏差 $(M e a n \pm S D)$ 表示。采用 SPSS17.0软件 (SPSS Inc., USA) 进行统计分析。用单因素 方差分析中Games-Howe11（方差非齐性）或Tukey（方差 齐性) 两两比较方法进行不同处理条件下的指标显著性差 异检验 $(P<0.05)$ 。

\section{3. 结果与讨论}

\section{1. 4 种光源对微藻光合放氧的影响}

光合放氧速率是植物光合强度的表征。为分析光源类 型及其光强变化对微藻光合器官功能的影响, 制作了 2 种 微藻在不同类型光源照射下的 $P-I$ 曲线, 见图 2 。在 4 种光 源下, 2 种微藻的 $P$ 值随 $I$ 的增强呈现出较为相似的变化规 律: 当I较低时, $P$ 值快速上升; 但是, 当 I高于某一值时, $P$ 值随光强的增强而降低, 即表现出光抑制效应。此时, 曲线上的转折点对应的光强即为微藻的光饱和点。根据光 抑制出现之前不同类型光源下的 $P$ 值变化, 4 种光源对微藻
的光合强度影响顺序如下: 普通小球藻为LED-W $>F L>$ LED-B $>$ LED-R; 盐生杜氏藻为LED-B $>$ FL $>$ LED-W $>$ LED-R。 根据 $P-I$ 曲线计算 2 株微藻在 4 种光源下的光合放氧参 数, 见表 2 。光源对微藻的 $\overline{P_{m}}$ 值和初始斜率 $S_{0}$ 有着不同的 影响, 普通小球藻和盐生杜氏藻的 $S_{0}$ 最大值分别出现在 $\mathrm{FL}$

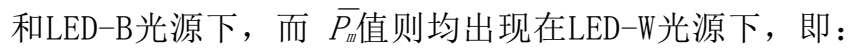
对于同一种微藻, 某种光源下的 $S$ 值最大, 其 $\overline{P_{m}}$ 值不一定 最高。普通小球藻和盐生杜氏藻在LED-W光源下获得的 $\overline{P_{m}}$ 最高值, 分别比FL照射下的 $\overline{P_{m}}$ 值提高 $71.39 \%$ 和 $2.90 \%$ 。这 表明, 与FL光源相比, LED-W照射能够一定程度上提高2 种微藻对无机碳的利用程度 [17], 从而合成更多的生物质, 并可进一步提升微藻的减排能力。

表2 通过 $P-I$ 曲线计算的微藻光合放氧参数。

\begin{tabular}{llcll}
\hline \multirow{2}{*}{ 微藻 } & \multirow{2}{*}{ 光源 } & \multicolumn{3}{c}{ 光合放氧参数 } \\
\cline { 3 - 5 } & & $\overline{\boldsymbol{P}_{\boldsymbol{m}}}$ & $\boldsymbol{S}_{\boldsymbol{o}}$ & $\overline{\boldsymbol{I}}_{\boldsymbol{m}}$ \\
\hline \multirow{4}{*}{ C. vulgaris } & LED-B & 407.25 & 2.45 & 45.14 \\
& LED-R & 286.58 & 2.65 & 68.68 \\
& LED-W & 529.80 & 4.35 & 47.94 \\
& $\mathrm{FL}$ & 309.12 & 8.92 & 47.35 \\
& LED-B & 436.83 & 11.93 & 64.73 \\
D. Salina & LED-R & 416.67 & 5.00 & 100.00 \\
& LED-W & 468.57 & 5.74 & 93.35 \\
& FL & 455.36 & 7.36 & 82.40 \\
\hline
\end{tabular}
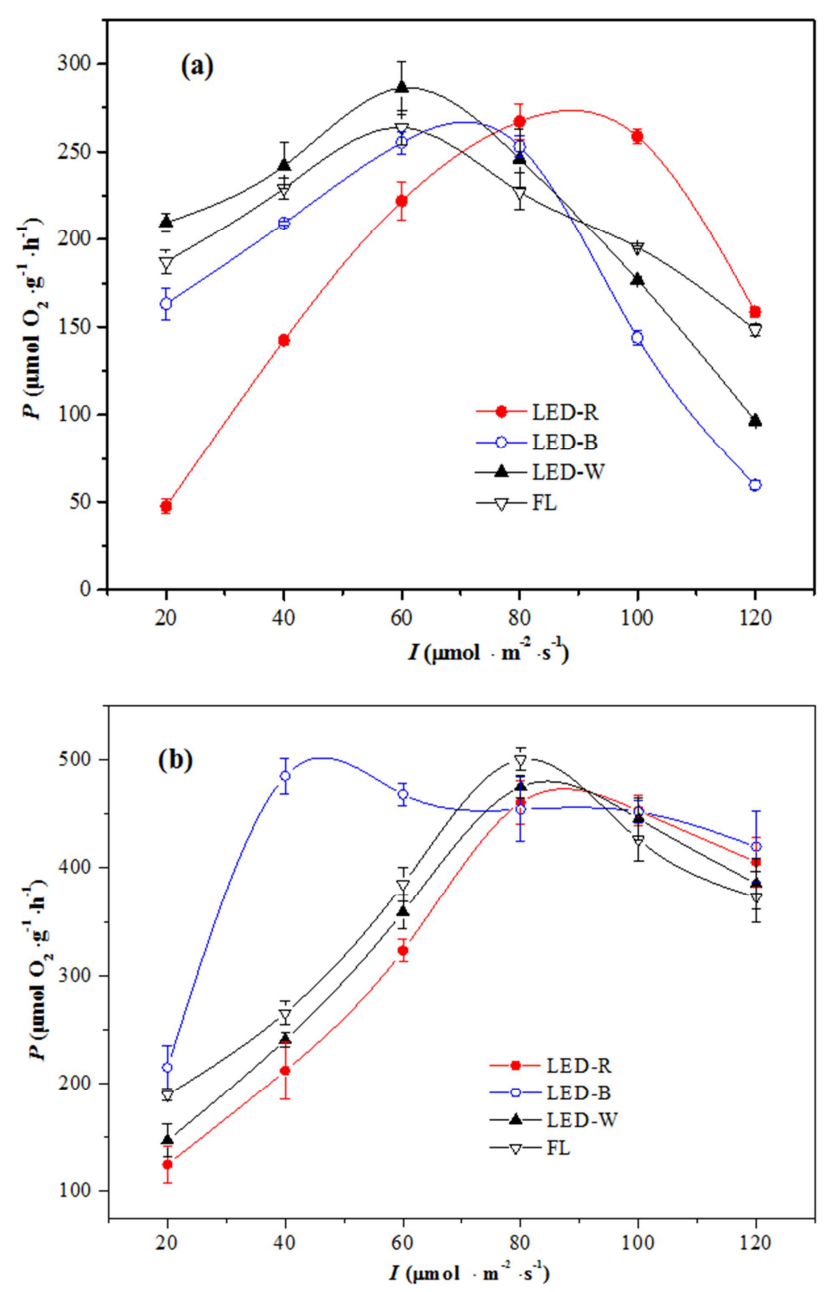

图2 微藻在 4 种光源下的 $P-I$ 曲线（a）普通小球藻;（b）盐生杜氏藻。 
目前, 国际上微藻培养所用的光源仍以 FL为主 （>95\%）[18]。作为日常照明光源, FL光源虽然具有节能、 发光效率高、光线柔和等优点, 但是, 将其作为微藻培养 的光源, 存在耗电量偏高、光谱针对性不强、几何形状单 一、易挥发录蒸气等缺点。LED被称为 “第四代光源”, 其在微藻培养中的应用于近几年受到了越来越多的关注。 主要原因为LED光源发射光谱能覆盖藻类生长所需的光谱 能带, 从而促进捕光合色素对光能的吸收, 提高藻生物量 与光合色素含量, 同时, 其能耗比 FL光源减少 $38.75 \%[18,19]$, 因此, 在微藻培养方面的应用潜力高于 FL。苗洪利等 [7] 以纤细角毛藻 (Chaetoceros gracilis) 和亚心形扁藻 (Platymonas subcordiformis) 为研究对象, 发现LED集成光源作为微藻生长的照明光源比荧光灯更有 优势。从本研究结果看, 虽然 4 种光源对光合强度的影响 顺序因藻种而异, 但是, 对于 2 种微藻而言, 至少有 1 种LED 光源对光合作用的促进效果优于FL光源, 表明某些LEDs 光源对微藻具有较好的促长效应。

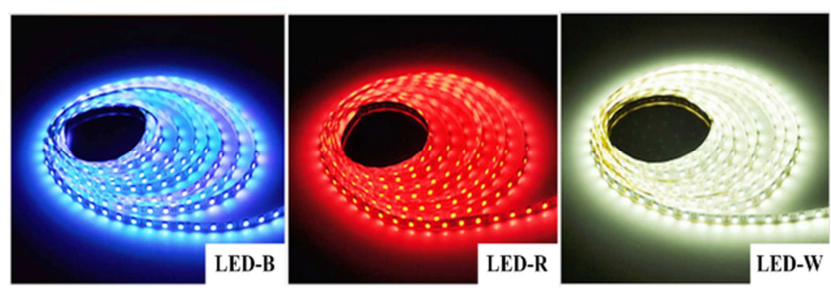

图3 不同颜色的LED条带灯。

\subsection{9 种光质对微藻生长的影响}

微藻在不同光质照射 (光强60 $\mathrm{\mu mol} \cdot \mathrm{m}^{-2} \cdot \mathrm{s}^{-1}$ ) 下的生 长曲线见图 4 。可以看出, 不同的光质条件, 微藻的生长 趋势存在很大差异。其中, 普通小球藻 (图4-a) 的生长 在经过 $2 \mathrm{~d}$ 的适应期后进入指数生长期, 至培养结束时, 以 $\mathrm{LW}+\mathrm{LB}$ 和 $\mathrm{LW}$ 照射下的藻生物量最高, 分别为 $0.19 \mathrm{~g} \cdot \mathrm{L}^{-1}$ 和 $0.18 \mathrm{~g} \cdot \mathrm{L}^{-1}$, 而 LR照射下的生物量最低。盐生杜氏藻（图 4-b) 则从第 $0 \mathrm{~d}$ 直接进入指数生长期, 至第 $6 \mathrm{~d}$ 仍未结束, 仅 $L W+L B 、 F W+L R 、 L R+L B 、 L R$ 等少数光质下出现生长速率 降低的现象; 与LR不同, 培养期间LB照射下的藻生物量一 直高于其他光质, 约为前者的 1.5 倍。

植物叶绿素的吸收波段主要是位于可见光区的蓝光 和红光, 因此, 微藻的比生长率受到光谱特征的影响。由 表3可见, 不同种类光质对微藻生长的影响程度因藻种而 异。9种光质下, 普通小球藻的 $\mu$ 值变化范围在 $0.11^{\sim} 0.22$ $\mathrm{d}^{-1}$, 最大 $\mu$ 值对应的光质为 $L W+L B$ 和 $L W$, 最低值出现在 LB+LR、LR照射下。但是, 同属绿藻门的盐生杜氏藻的 $\mu$ 值范围为 $0.41^{\sim} 0.44 \mathrm{~d}^{-1}$, 高 $\mu$ 值出现在LB、FW、FW+LR三 种光质下, 最低 $\mu$ 值出现在LR光质下。前人的研究也观察 到, 绿藻门中不同藻种的最适宜光质不同。毛安君等 [20] 研究发现, 直接用LED提供单色光质时, 小球藻在LB下的 生物量最大; 而Yan等 [21] 认为 LWW最适于小球藻生长; 栅 藻 (Scenedesmus sp. LX-1) [22] 和亚心形扁藻 [7] 却在 $L R+L B$ 下生长最快。一般认为, 入射光的波长应与光合色 素的吸收光谱相一致, 本研究观察到 2 种微藻的比生长速
率对同种光质的响应存在差异, 这可能与其光合生产中各 自需要吸收不同波长的光有关。
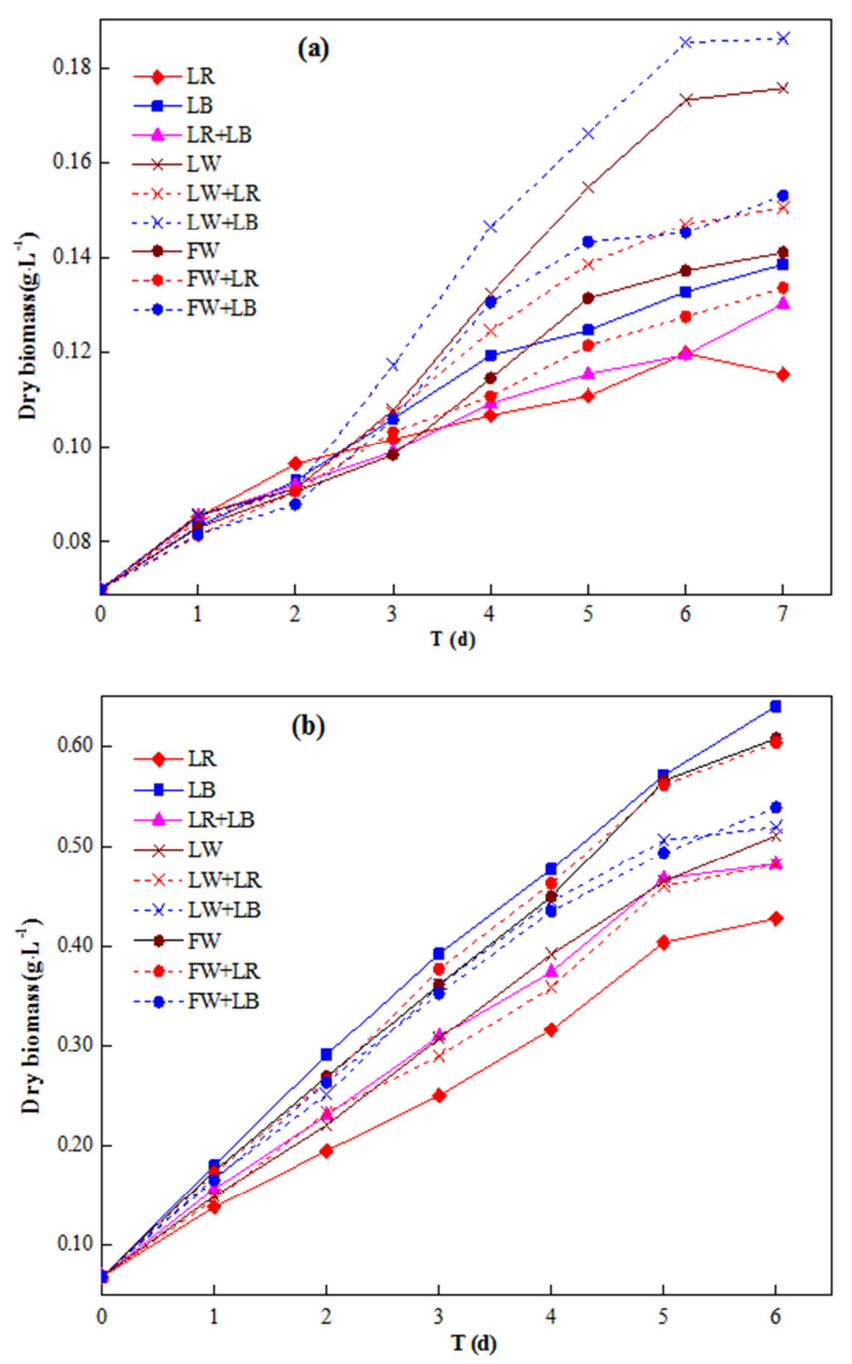

图4 2种微藻在不同光质中的生长曲线（a）普通小球藻; (b) 盐生杜氏 藻。

\section{3. 不同光质照射下微藻的光合色素含量}

叶绿素是微藻光合作用的天线色素, 参与光能的吸收 和传递。绿藻中主要为 Ch1 $-a$ 和Ch1- $b$, 其吸收光谱在红 光区和蓝光区各有一个吸收峰, 位于波长 $435 \mathrm{~nm} 、 676 \mathrm{~nm}$ 处 [23]。此外, 绿藻的另一种辅助色素是Car, 主要吸收 光谱中的蓝光 $\left(400^{\sim} 550 \mathrm{~nm}\right)$, 主要功能有两个: 一是在合 成途径中产生的分子 (如 $\beta$-胡萝卜素, 叶黄素) 被运输 到细胞内脂质体, 脂质体分散在外围可以作为遮光剂, 减 少过剩的光照对叶绿体活性的影响 [24]; 二是通过扩展微 藻的吸收光谱以增加光能利用效率 [23]。

微藻光合作用中, PII和PSI由捕光色素蛋白复合体和 反应中心色素蛋白复合体组成, 捕光色素包括Ch1-a、 Ch1-b、Car等; 而反应中心色素主要为叶绿素分子P680 和P700; 捕光色素的吸收光谱在红光区和蓝光区各有一个 吸收峰, 意味着色素更容易吸收这两个波段的光, 跃迁至 第一单线态和第二单线态, 继而推动整个非环式电子传递 过程 [25]。其中, 吸收蓝光的第二单线态有更多的能量用 
于光合作用, 而吸收红光的第一单线态除进行光合作用外, 还会发出菼光甚至回落至一三线态后发出磷光, 能量更少, 这可能是LB比LR更有利于微藻生长的原因之一。由 3.2 节 可知, 叶绿素对不同种类光质的吸收存在差异, 微藻表现 出不同的比生长率。但是, 这种差异是否能够通过影响光 合色素含量而实现尚不明确。因此, 本研究分别测定了 2 种微藻在不同光质下的光合色素含量, 结果列于表4。

表3 2种微藻在不同光质下指数生长期的比生长速率。

\begin{tabular}{lll}
\hline \multirow{2}{*}{ 光质 } & \multicolumn{2}{c}{$\mu\left(\mathrm{d}^{-1}\right) *$} \\
\cline { 2 - 3 } & C. vulgaris & D. salina \\
\hline LR & $0.11 \pm 0.0042^{\mathbf{a}}$ & $0.40 \pm 0.0004^{\mathbf{a}}$ \\
LB & $0.15 \pm 0.0014^{\mathbf{b}}$ & $0.44 \pm 0.0004^{\mathbf{d}}$ \\
LR+LB & $0.12 \pm 0.0007^{\mathbf{a}}$ & $0.41 \pm 0.0016^{\mathbf{b}}$ \\
LW & $0.20 \pm 0.0024^{\text {de }}$ & $0.42 \pm 0.0020^{\mathbf{c}}$ \\
LW+LR & $0.17 \pm 0.0016^{\mathbf{c}}$ & $0.41 \pm 0.0085^{\text {bc }}$ \\
LW+LB & $0.22 \pm 0.0010^{\mathbf{e}}$ & $0.42 \pm 0.0042^{\mathbf{c}}$ \\
FW & $0.16 \pm 0.0019^{\mathbf{f}}$ & $0.44 \pm 0.0010^{\mathbf{d}}$ \\
FW+LR & $0.14 \pm 0.0022^{\mathbf{z}}$ & $0.44 \pm 0.0032^{\mathbf{d}}$ \\
FW+LB & $0.18 \pm 0.0018^{\mathbf{h}}$ & $0.42 \pm 0.0028^{\mathbf{c}}$ \\
\hline
\end{tabular}

* 字母不同表示 $\mu$ 有显著性差异 $(P<0.05)$

结果如下: 对普通小球藻而言, 在 9 种光质照射下, 以复合光谱 $\mathrm{FW}+\mathrm{LR}$ 的色素含量最高, Ch1-a、Ch1-c、Car 含量分别为 $9.87,3.66,4.90 \mathrm{mg} \cdot \mathrm{L}^{-1}$; 明显高于 $F W$ 照射下 的色素含量, 表明在连续光谱基础上加大红光比率, 有利 于光合色素合成。Mohsenpour等[26]用150 W的氙弧灯模 拟阳光, 照射含紫、绿、橙、红色染料的亚克力板条产生 所需波长的光, 作为普通小球藻生长的光源, 培养 $14 \mathrm{~d}$ 后, 最大Ch1- $a$ 含量发生于红光照射下，并认为所提供的 红光波段 $\left(650^{\sim} 700 \mathrm{~nm}\right)$ 是 $\mathrm{Ch} 1-a$ 的吸收光谱, 因而对小 球藻色素合成起促进作用。但是, 本研究发现蓝光在其中 也起到重要作用, 表现为 $L W+L R$ 的色素含量显著低于 $L W$ 、 LB、 $L W+L B$ 以及 $L R$ 与 $L B$ 照射下的色素含量差异不明显。这
些现象说明, LR对色素合成的促进作用需要其他波段的光 （尤其是LB）的参与 [26]。在表4中，LR对色素积累的提 升作用在盐生杜氏藻中较为明显, 这与 $\mathrm{Sa}^{\prime}$ nchez-Saavedra等[27]对盐藻的研究结果一致。本研 究中, 盐藻光合色素含量对光质的响应为: LR下 3 种色素 含量均最高 (Ch1- $a 、$ Ch1- $c$ 、Car含量分别为21. 14, 6.60, $\left.10.10 \mathrm{mg} \cdot \mathrm{L}^{-1}\right)$ ，尽管与 $\mathrm{LW} 、 \mathrm{LW}+\mathrm{LR}$ 照射下差异不显著, 但 明显高于 $L B 、 L W+L B$ 组; 在 $F W 、 F W+L R 、 F W+L B$ 三种光质中, 色素含量也以FW+LR为高。与LR相比, LB对盐生杜氏藻的 光合色素含量的调控作用不明显。综合以上结果发现, 载 复合光谱基础上加大LR比例有利于提高普通小球藻与盐 生杜氏藻的色素含量, 而在单色光质LR、LB下色素含量往 往较低。可能的原因是, 微藻光合色素的合成需要多种光 质的协同参与 [28], 且红光是PSII和PSI优先吸收的光质 [29]，这二者的作用共同引起了该现象。

\section{4. 光质变化调控微藻生长的可能原因}

对 2 种微藻在不同光质下的 $\mu$ 值与光合色素含量进行 相关性检验, 结果见表5。2种微藻的光合色素含量与 $\mu$ 值 均不相关, 即: 随光质种类的变化, 微藻的光合色素含量 与 $\mu$ 值表现出非一致性的响应特征。由此推断, 光合色素 变化可能不是光质调控微藻生长的主要原因, 尚存在其他 影响微藻的光能利用的机制。

图5为本研究所用 2 种微藻的活体吸收谱, 它们在蓝光 区和红光区均有吸收峰, 分别位于 $430 \mathrm{~nm}$ 左右和 $680 \mathrm{~nm}$ 左右, 与Mouget等 [28] 的报道类似。已有研究发现, 微藻 生长与比消光系数之间存在较好的一致性 [30]。消光系数 与入射光光强无关, 表明同样光量子通量密度下, 藻细胞 会吸收更多的蓝光或红光光能, 这可能是LB或LR促进微藻 生长的另一个原因。比较而言, 普通小球藻对LB的吸收系 数明显高于 $\mathrm{LR}$, 而盐生杜氏藻对 $\mathrm{LB}$ 与 LR的吸收系数相近, 因此在两种单色光下的盐藻 $\mu$ 接近。

表4 光强60 $\mu \mathrm{mo} 1 \cdot \mathrm{m}^{-2} \cdot \mathrm{s}^{-1}$ 的 9 种光质下 2 种微藻的光合色素含量。

\begin{tabular}{|c|c|c|c|c|c|c|}
\hline \multirow{3}{*}{ 光质 } & \multicolumn{6}{|c|}{ 色素含量 $\left(\mathrm{mg} \cdot \mathrm{L}^{-1}\right) *$} \\
\hline & \multicolumn{3}{|c|}{ C. vulgaris } & \multicolumn{3}{|c|}{ D. salina } \\
\hline & Ch1-a & Ch1-b & Car & Ch1-a & Ch1-b & Car \\
\hline LR & $8.04 \pm 0.06^{b}$ & $2.95 \pm 0.32^{\mathrm{ab}}$ & $4.09 \pm 0.27^{b}$ & $21.14 \pm 0.14^{\mathrm{d}}$ & $6.60 \pm 0.18^{\mathrm{d}}$ & $10.10 \pm 0.10^{c}$ \\
\hline LB & $8.56 \pm 0.21^{b}$ & $3.07 \pm 0.29^{\mathrm{ab}}$ & $4.60 \pm 0.20^{c}$ & $16.28 \pm 0.70^{\mathrm{bc}}$ & $4.21 \pm 0.11^{\mathrm{b}}$ & $7.75 \pm 0.40^{\mathrm{b}}$ \\
\hline $\mathrm{LR}+\mathrm{LB}$ & $8.59 \pm 0.31^{\mathrm{b}}$ & $3.13 \pm 0.19^{\mathrm{abc}}$ & $4.48 \pm 0.16^{\mathrm{bc}}$ & $17.74 \pm 0.24^{c}$ & $4.93 \pm 0.30^{\mathrm{bc}}$ & $8.38 \pm 0.10^{b}$ \\
\hline $\mathrm{LW}$ & $9.61 \pm 0.34^{\text {cd }}$ & $3.53 \pm 0.23^{\mathrm{bc}}$ & $4.84 \pm 0.06^{c}$ & $20.39 \pm 1.06^{\mathrm{d}}$ & $5.89 \pm 0.65^{\text {cd }}$ & $9.54 \pm 0.44^{c}$ \\
\hline $\mathrm{LW+LR}$ & $6.99 \pm 0.15^{\mathrm{a}}$ & $2.68 \pm 0.23^{\mathrm{a}}$ & $3.58 \pm 0.13^{\mathrm{a}}$ & $20.53 \pm 1.03^{\mathrm{d}}$ & $6.07 \pm 0.15^{\mathrm{d}}$ & $9.86 \pm 0.63^{\mathrm{c}}$ \\
\hline $\mathrm{LW+LB}$ & $8.43 \pm 0.26^{\mathrm{b}}$ & $3.07 \pm 0.07^{\mathrm{ab}}$ & $4.62 \pm 0.18^{c}$ & $13.29 \pm 0.39^{\mathrm{a}}$ & $3.05 \pm 0.07^{\mathrm{a}}$ & $6.45 \pm 0.35^{\mathrm{a}}$ \\
\hline FW & $8.73 \pm 0.25^{\mathrm{bc}}$ & $3.18 \pm 0.09^{\mathrm{ab}}$ & $4.42 \pm 0.15^{\mathrm{bc}}$ & $15.22 \pm 1.02^{\mathrm{ab}}$ & 4. $03 \pm 0.10^{\mathrm{b}}$ & $7.24 \pm 0.64^{\mathrm{ab}}$ \\
\hline $\mathrm{FW}+\mathrm{LR}$ & $9.87 \pm 0.50^{d}$ & $3.66 \pm 0.10^{c}$ & $4.90 \pm 0.04^{c}$ & $16.06 \pm 0.48^{\mathrm{bc}}$ & $4.16 \pm 0.64^{\mathrm{b}}$ & $7.76 \pm 0.02^{b}$ \\
\hline $\mathrm{FW}+\mathrm{LB}$ & $8.66 \pm 0.48^{\mathrm{bc}}$ & $3.09 \pm 0.07^{\mathrm{abc}}$ & 4. $75 \pm 0.21^{c}$ & $15.48 \pm 0.25^{\mathrm{b}}$ & $4.09 \pm 0.18^{b}$ & $7.28 \pm 0.36^{\mathrm{ab}}$ \\
\hline
\end{tabular}

* 字母不同表示色素含量有显著性差异 $(P<0.05)$

表5 2种微藻比生长速率与色素含量间的相关性系数。

\begin{tabular}{cccc}
\hline 微藻 & \multicolumn{3}{c}{ 色素含量与 $\mu$ 之间的相关系数 } \\
\hline \multirow{2}{*}{ C. vulgaris } & Ch1-a & Ch1-b & CD \\
& 0.0776 & 0.0702 & 0.2447 \\
D. salina & Ch1-a & Ch1-b & CD \\
& -0.6137 & -0.6458 & -0.6174 \\
\hline
\end{tabular}



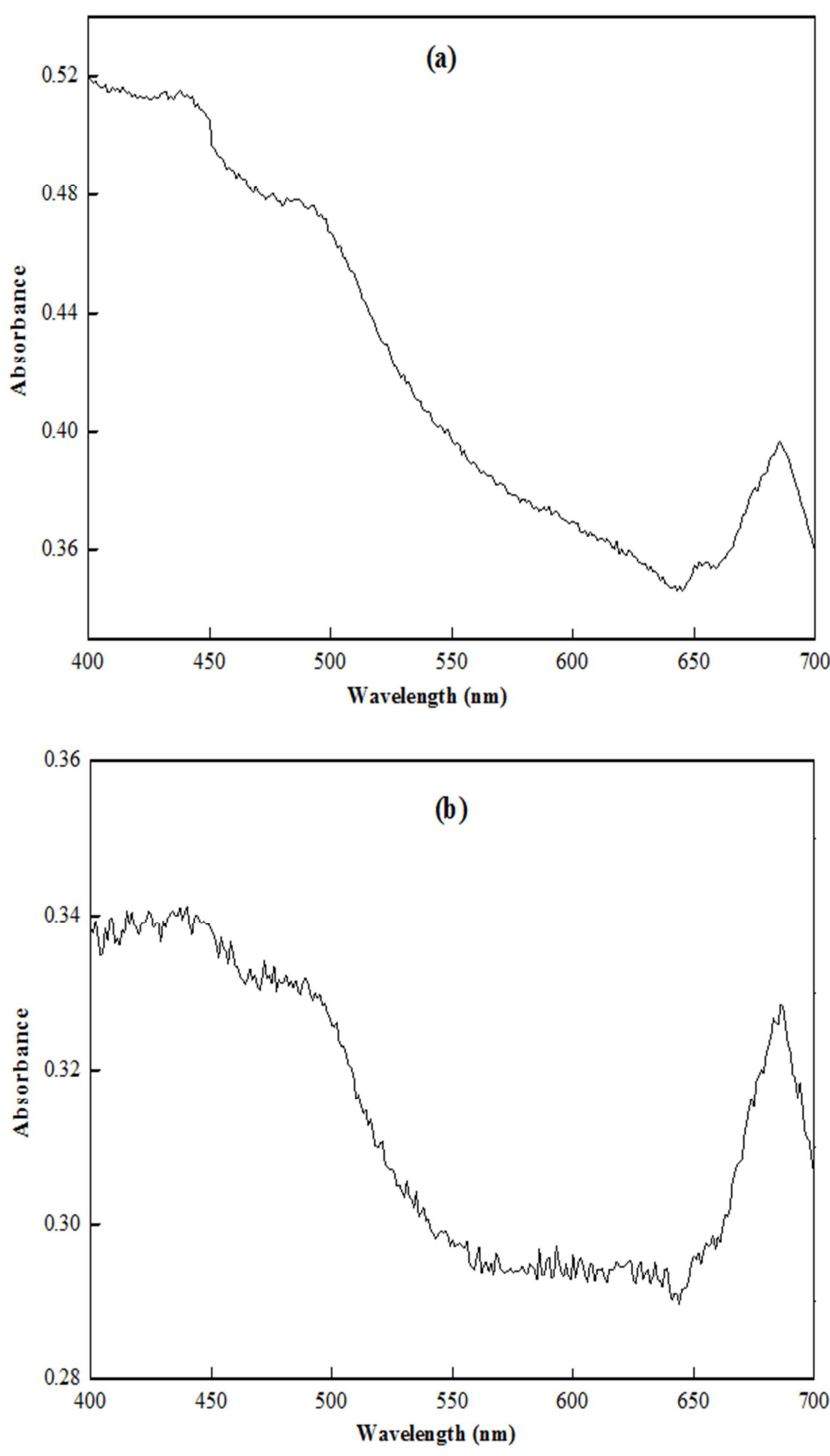

图5 2种微藻在PAR区的活体吸收谱。

\section{4. 结论}

(1) 用 4 种人工光源对微藻进行培养, 普通小球藻、盐 生杜氏藻的最大光合放氧速率均出现在LED-W照射下。 LEDs对微藻光合作用的促进效果优于FL光源。(2) 适宜微 藻生长的光质条件存在种质差异性: 普通小球藻的最适光 质为 $\mathrm{LW}+\mathrm{LB}$ 或 $\mathrm{LW}$; 而盐生杜氏藻生长受光质调控不明显。

(3) 光合色素含量与 $\mu$ 值随光质变化的非一致性说明光合 色素变化可能不是光质调控微藻生长的主要原因。藻细胞 的活体光吸收可能是影响微藻生长的主要因素。

\section{致谢}

本文为国家科技支撑计划课题 (2011BAD14B04) 资助 的阶段性成果之一。

\section{参考文献}

［1］李永富, 孟范平, 李祥蕾等. 光照对光生物反应器中微藻高 密度光自养培养的影响 $[\mathrm{J}]$. 中国生物工程杂 质, 2013, 33(2) : 103-110。

[2] Ugwu C U, Aoyagi H, Uchiyama H. Photobioreactors for masscultivation of algae $[\mathrm{J}]$. Bioresource Technology, 2008, 99 (10) : 4021-4028.

［3］李元广, 谭天伟, 黄英明. 微藻生物柴油产业化技术中的若 干科学问题及其分析 [J]. 中国基础科学, 2009 (5) :64-70。

[4] Das P, Wang L, Aziz S S, et al. Enhanced algae growth in both phototrophic and mixotrophic culture under blue light [J]. Bioresource Technology, 2001, 102 (4) : 3883-3887.

［5］郝洛西, 杨秀. 基于LED光源特性的半导体照明应用创新与 发展 [J]. 照明工程学报, 2012, 23(1) : 1-6。

[6] Katsuda T, Shimahara K, Shiraishi H, et al. Effect of flashinglight from blue light emitting diodes on cell growth and astaxanthin production of Haematococcus pluvialis [J]. Journal of Bioscience and Bioengineering, 2006, $102(5)$ : 442-446.

[7] 苗洪利, 周晓光, 刘逢学, 等. LED光谱对纤细角毛藻和亚心 形扁藻生长的影响 [J]. 光学学报, 2010, 30 (4) : 1101-1105。

［8］唐青青, 方治国, 秡雯雯, 等. 光质对蛋白核小球藻 (Chlorella pyrenoidosa) 生长特征及生化组成的影响研 究 [J]. 环境科学, 2014, 35 (11) : 4212-4217。

[9］苗洪利, 孙丽娜, 田庆震, 等. LED单色光谱及复合光谱对赤 潮优势种中胁骨条藻生长的作用 $[\mathrm{J}]$. 中国海洋大学学报, 2011, 41 (10): 107-110。

[10] 王伟. 光质对中华盒形藻生长及生化组成的影响 [J]. 武汉 植物学研究, 1999, 17 (3) : 197-200。

[11] 沈银武, 朱运芝, 刘永定. 不同光质对中华植生藻的影响 $[\mathrm{J}]$. 水生生物学报, 1999, 23 (3) :285-287。

[12] Han B P. A mechanistic model of algal photoinhibition induced by photodamage to photosystem-II [J]. Journal of Theoretical Biology, 2002, 214(4) : 519-527.

[13] Ge Y, Liu J, Tian G. Growth characteristics of Botryococcus brauni 765 under high $\mathrm{CO}_{2}$ concentration in photobioreactor [J]. Bioresource Technology, 2011, $102(1)$ : 130-134.

[14] Sükran D, Günes T, Sivaci R. Spectrophotometric determination of chlorophyll-A, B and total carotenoid contents of some algae species using different solvents [J]. Turkish Journal of Botany, 1998, 22: 13-17. 
[15] Ritchie R J. Universal chlorophyll equations for estimating chlorophylls a, b, c, and d and total chlorophylls in natural assemblages of photosynthetic organisms using acetone, methanol, or ethanol solvents [J]. Photosynthetica, 2008, 46(1): 115-126.

[16] Jensen A. Chlorophylls and carotenoids [J]. In: Handbook of phycological methods. Cambridge University Press, London, 1978, pp 59-70.

[17] 许博, 周斌, 鞠青, 等. 海洋微藻光合作用对 $\mathrm{CO}_{2}$ 加富的响应 特征 $[J]$. 海洋环境科学, 2010, 29 (006) : 790-793。

[18] 毛安君. LED光源促进微藻生长的研究 [D]. 青岛: 中国海洋 大学, 2006。

[19] Chen C Y, Yeh K L, Aisyah R, et al. Cultivation, photobioreactor design and harvesting of microalgae for biodiesel production: A critical review [J]. Bioresource Technology, 2011, 102(1):71-81.

[20] 毛安君, 王晶, 林学政, 等. 光谱对小球藻和等鞭金藻生长的 影响 [J]. 光谱学与光谱分析, 2008, 28 (5) : 991-994。

[21] Yan C, Zhang L, Luo X, et al. Effects of various LED light wavelengths and intensities on the performance of purifying synthetic domestic sewage by microalgae at different influent $\mathrm{C} / \mathrm{N}$ ratios $[\mathrm{J}]$. Ecological Engineering, 2013, 51: 24-32.

[22] 李金鏨, 洪营, 杨佳. LED红光/蓝光对栅藻LX1生长及产油特 性的影响 [J]. 环境科学, 2010, 31 (2) :244-250。

[23] Lee E, Heng R L, Pilon L. Spectral optical properties of selected photosynthetic microalgae producing biofuels [J]. Journal of Quantitative Spectroscopy and Radiative Transfer, 2013, 114: 122-135.

[24] Zhekisheva M, Boussiba S, Khozin - Goldberg I, et al. Accumulation of oleic acid in Haematococcus pluvialis (chlorophyceae) under nitrogen starvation or high light is correlated with that of astaxanthin esters 1 [J]. Journal of Phycology, 2002, 38(2) : 325-331.

[25] 韩博平, 韩志国, 付翔. 藻类光合作用机理与模型 [M]. 北京: 科学出版社, 2003。

[26] Mohsenpour S F, Richards B, Willoughby N. Spectral conversion of light for enhanced microalgae growth rates and photosynthetic pigment production [J]. Bioresource Technology, 2012, 125: 75-81.

[27] Sánchez-Saavedra M P, Jiménez C, Figueroa F L. Variable fluorescence of chlorophyll a in Dunaliella bardawil with different $\beta$-carotene content $[\mathrm{J}]$. Scientia Marina, 1996, 60(1): 227-231.

[28] Mouget J L, Rosa P, Tremblin G. Acclimation of Haslea ostrearia to light of different spectral qualities confirmation of 'chromatic adaptation' in diatoms [J]. Journal of Photochemistry and Photobiology B: Biology, 2004, 75(1) : 1-11.

[29] 于贵瑞, 王秋风. 植物光合、蒸腾与水分利用的生理生态学 [M]. 北京: 科学出版社, 2010。

[30] 刘春朝, 刘瑞, 王锋. 微藻培养过程的光特性研究进展 [J]. 生物加工工程, 2011, 9(6):69-76。 